\title{
LETTERS
}

doi:10.1017/\$1041610209991499

\section{Antipsychotics in dementia: are they being appropriately prescribed?}

In 2007, the U.K.'s All-Party Parliamentary Group on Dementia (APPG) undertook an inquiry into the prescription of antipsychotics for people with dementia on account of concerns expressed by carers, patient organizations and academics about the appropriateness and safety of these drugs (AllParty Parliamentary Group on Dementia, 2008). It has been estimated that $32 \%$ of patients with dementia in care homes are prescribed antipsychotics (Alldred et al., 2007). Both typical and atypical antipsychotics are associated with QT prolongation, which can lead to arrhythmias and sudden death (Joint Formulary Committee, 2009). They are also associated with an increased risk of cerebrovascular events. Adverse effects in the elderly include excessive sedation and dizziness, which can lead to falls and therefore accelerated cognitive decline.

Behavioral and psychological symptoms (BPSD) are common in dementia, occurring in up to $80 \%$ of cases (Ballard et al., 2008). They include agitation, aggression, delusions, hallucinations, disinhibition, irritability and aberrant motor behavior.

NICE guidelines state that antipsychotics should only be considered for severe or agitated behavior causing significant distress or if there is immediate risk of harm to self or others (National Institute for Health and Clinical Excellence, 2006). It is therefore pertinent to discuss the anticipated use of these drugs with patients and their carers. Target symptoms should be identified and documented and the subsequent change in these symptoms should be assessed and recorded regularly. Treatment with antipsychotics should be time-limited and reviewed every three months or according to clinical need. In 2008, the APPG acknowledged the excessive prescription of antipsychotics and stressed the importance of adhering to NICE guidelines regarding the prescription of antipsychotics. They also highlighted the need to audit the prescription of antipsychotics in patients with dementia.

We identified patients referred to an Old Age Psychiatric team based at The Queen Elizabeth Psychiatric Hospital in Birmingham, U.K., using medical and electronic records. All patients seen between August 2005 and August 2007 who received a DSM-IV (American Psychiatric Association, 1994) diagnosis of dementia were included in our audit. Medical notes were reviewed to obtain: age; gender; type of dementia; MMSE score; presenting symptoms; presence of BPSD; treatment offered, including antipsychotics; duration of pharmacological treatment; mean follow-up.

Seventy patients were identified. The study population comprised 27 men and 43 women, with a median age of 76 years. Thirteen $(18.6 \%)$ patients had mild cognitive impairment, $51(72.9 \%)$ moderate cognitive impairment and $6(8.6 \%)$ severe cognitive impairment. Fifty $(71.4 \%)$ had BPSD and antipsychotic medication was prescribed in $14(28.0 \%)$ of these. Of these $14,11(78.6 \%)$ received typical antipsychotics and 3 (21.4\%) atypical antipsychotics.

Of the 14 patients prescribed antipsychotics, six $(42.9 \%)$ were prescribed these drugs for less than three months, three $(21.4 \%)$ for three to six months, and five $(35.7 \%)$ for more than six months. The maximum period for which any patient took antipsychotics was eight months. The remaining 36 patients with BPSD were not prescribed antipsychotics.

A significant number of patients with dementia who were seen by this Old Age Psychiatric service presented with BPSD. The majority of these were not treated with antipsychotics. This Old Age Psychiatric team is adhering to NICE guidelines: namely, the use of antipsychotics is limited to patients with severe BPSD, and antipsychotics are prescribed to most patients for a limited period of time.

From our audit findings we believe that clinicians would benefit from adopting the following guidance:

- The risks and benefits of prescribing antipsychotics should be reviewed prior to prescribing antipsychotics in patients with dementia.

- Teams should document the BPSD for which antipsychotics are prescribed.

- Teams should document discussions with patients and their carers about antipsychotic prescriptions.

- A flagging system should be introduced to remind clinicians to review antipsychotic prescriptions and symptoms in patients with dementia every three months.

- Clinical teams should regularly audit their prescribing of antipsychotics in patients with dementia.

Whilst there is much evidence regarding the potential adverse effects of antipsychotics, their benefit in relieving symptoms in patients with dementia is also clear. The findings of the APPG have highlighted the need for regulatory processes 
to ensure these drugs are used in an appropriate and safe manner and audit is a simple and effective means of achieving this.

\section{Conflict of interest declaration}

AP is the consultant of the Old Age Psychiatric team whose performance we audited.

\section{References}

All-Party Parliamentary Group on Dementia (2008) Always a Last Resort. Inquiry into the Prescription of Antipsychotic Drugs to People with Dementia Living in Care Homes. London: Alzheimer's Society.

Alldred, D. P. et al. (2007). Antipsychotic prescribing patterns in care homes and relationship with dementia. Psychiatric Bulletin, 31, 329-332.

American Psychiatric Association (1994). Diagnostic and Statistical Manual of Mental Disorders, 4th edn. Washington, DC: American Psychiatric Association.

doi:10.1017/\$1041610209991700

\section{Old age psychiatry and geriatric medicine admissions and elderly suicide rates in England}

Almost all elderly suicide victims have mental illness, and up to $90 \%$ have depression (Shah and De, 1998). A significant number of elderly suicide victims in Western countries consult their general practitioner or psychiatrist or contact mental health services between one week and six months prior to the suicide (Catell, 1988; Conwell et al., 1990; 1991; Catell and Jolley, 1995; Vassilas and Morgan, 1993; 1994). This offers an opportunity for identification and treatment of the mental illness. Thus, the availability of appropriate healthcare services may be an important factor associated with elderly suicide rates.

High suicide rates in China (both rural areas and Hong Kong), in part, have been attributed to lack of mental health services and poorly developed social welfare and benefit systems (Yip and Tan, 1998; Jianlin, 2000; Yip, 2001; Yip et al., 2000). Moreover, reduction in elderly suicide rates in Singapore and urban China have, in part, been attributed to improved healthcare and welfare services for the elderly (Kua et al., 2003; Yip et al., 2005). A decline in elderly suicide rates in England and Wales was associated with measures of improved healthcare for the elderly (Gunnell et al., 2003; Lodhi and Shah, 2005) including an increase in the number of general practitioners, hospital medical staff, outpatient appointments for mental
Ballard, C. et al. (2008). Neuropsychiatric symptoms in dementia: importance and treatment considerations. International Review of Psychiatry, 20, 396-404.

Joint Formulary Committee (2009). British National Formulary, 57th edn. London: British Medical Association and Royal Pharmaceutical Society of Great Britain.

National Institute for Health and Clinical Excellence (2006). Dementia: Supporting People with Dementia and their Carers in Health and Social Care. Available at: http://www. nice.org.uk/nicemedia/pdf/CG042NICEGuideline.pdf.

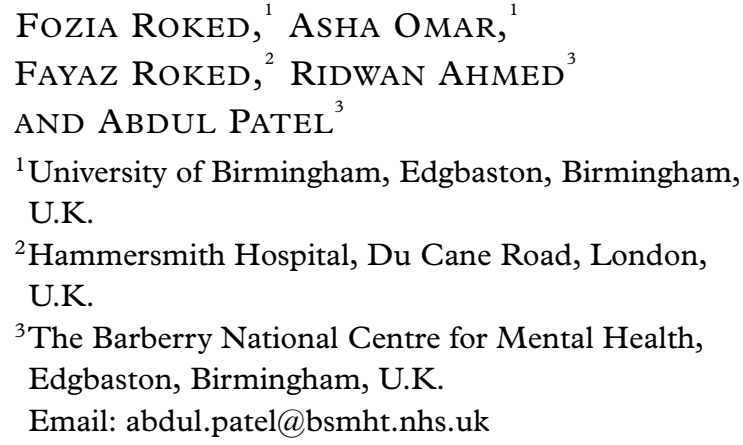

illness, and field social workers and day center staff (Lodhi and Shah, 2005). However, in a crossnational study elderly suicide rates were higher in countries with greater numbers of psychiatric beds, psychiatrists and psychiatric nurses (Shah and Bhat, 2008). The relationship between elderly suicide rates and the utility of inpatient old age psychiatry (OAP) services has not been examined. Therefore, a study examining the relationship between elderly suicide rates and the utility of inpatient geriatric medicine (GM) and OAP services in England was undertaken with the null hypothesis that there will be no significant relationship between these variables. GM was considered because a third of GM inpatients have depression (Shah and Hoxey, 2000) and suicidal ideation (Shah et al., 2000).

Data on suicide rates in both sexes in the age-bands 65-74 years and 75+ years for England and Wales was ascertained from the World Health Organization (WHO) website (http://www.who.int/ whosis/database/mort/table1.cfm) for each of the nine calendar years between 1998 and 2006. Data on the number of finished episodes (i.e. the number of patients discharged with a completed inpatient episode), median length of stay, and the number of bed days for patients admitted to GM services and OAP services in England was ascertained from nationally collected data (Hospital Episode Statistics, available at: www.hesonline.nhs.uk/Ease/ servlet/ContentServer?siteID=1937\&CategoryID= 192). These national data are gathered centrally via the patient administration systems in nearly 400 hospitals in England. Data were ascertained for the 